\title{
Efficient search of obstacle-free paths for anthropomorphic hands
}

\author{
R. Suárez, J. Rosell, A. Pérez and C. Rosales
}

\begin{abstract}
The planning of collision-free motions of a handarm system to reach a grasp or preshape configuration is not a simple issue due to the high number of involved degrees of freedom. This paper presents an efficient sampling-based path planner that copes with this issue by considering a reduced search space. The dimension of this space is not fixed but it is iteratively increased according to the difficulty of the task at hand. Initially the search space is 1-dimensional along the line defined by the initial and goal hand configurations (by construction those configurations always belong to the search space), and then its dimension is increased by iteratively adding principal motion directions (that couple the finger motions), trying in this way to produce hand movements through anthropomorphic natural postures.
\end{abstract}

\section{INTRODUCTION}

A great amount of work is currently dedicated to humanlike robots, ranging from aspects like the robot appearance and expression to the mimicking of the human movements in order to perform a given task. Some basic problems have to be solved in this line, being one of them the coordination of movements in a system with a large number of degrees of freedom, for instance to allow the robot walking or to manipulate objects. The latter involves the coordination of the hand-arm system, since most of the tasks that a robot is expected to do include interaction with the environment, either grasping an object and moving it to another place or doing some particular work with it. Solving this problem requires the planning of the movements of the set handarm in order to find a valid trajectory, i.e. a valid path in the joint coordinates and a temporal evolution of the movement of each of them. This problem can be formulated as a typical motion planning problem in a $n$-dimensional configuration space [1], which may allow optimal solutions according to predefined criteria, but the computational cost is really high. In order to reduce the computational cost and look for more practical solutions some know-how about the reachable space and basic movements of an anthropomorphic hand should not be missed, and some reduction of the search space should likely be done, but without losing the humanlike appearance of the movements. This work deals with this problem, proposing an approach that initially considers the movements of the arm and the movements of the hand separately, and then looks for a common solution using a unique probabilistic roadmap [2], moreover, the search space

This work was partially supported by the Spanish Government through the projects DPI2007-63665 and DPI2008-02448.

The authors are with the Institute of Industrial and Control Engineering, Technical University of Catalonia, Barcelona, Spain. C. Rosales is also with the Institut de Robòtica i Informàtica Industrial, CSIC-UPC. A. Pérez is also with the Escuela Colombiana de Ingeniería "Julio Garavito", Bogotá D.C., Colombia. Contact: raul.suarez@upc.edu is initially considered of dimension 1 , and the dimension is then progressively increased if no solution is found.

The problem of planning the hand-arm movements was already tackled considering a search-space with a reduced dimension in [3]. In that work only a few principal motions directions of the hand are used to find a collision free path for the hand-arm, but this constraint in the potential movements of the hand limits its dexterity in complex environments (i.e. those with several and/or complicate obstacles) and generates the need for special movements at the initial and goal states if they do not belong to the (pre)selected search space. The approach presented in this work avoids these problems allowing an incremental hand dexterity, starting from a simple direct path and arriving to the complete model of the real problem if a solution is not found on the way.

\section{BASIC BACKGROUND}

\section{A. Principal Motion Directions}

Principal Motion Directions (PMDs) are basically coordinated movements of the hand joints. They can be used to reduce the problem of planning the motion of a mechanical hand, considering that the human hand also has this couplings. They are obtained by taking samples of human hand postures using a sensorized glove, mapping them to the mechanical hand and then performing a principal component analysis (PCA) [4] over the set of samples. The samples are taken with the intention of covering the mechanical hand workspace. It is worth to note that this mapping is critical to achieve such a goal, thus the more anthropomorphic the mechanical hand is, the easier the mapping results.

In this work, the Schunk Antropomorphic Hand (SAH) [5] is used. It has four identical fingers and one is equipped with an additional joint to function as the opposing thumb. Each finger has four joints, one for abduction and three for flexion, with two of them coupled, having therefore three degrees of freedom (DOF) per finger. In total, it has 13 DOF. Nevertheless, the samples are 11-dimensional due to the mapping from the glove to the hand, since some joints were attached to a single sensor to give a better immersion to the user while taking the samples (details on this mapping can be found in [3]).

The PCA involves the computation of the eigenvalue decomposition of a data covariance matrix or the singular value decomposition of a data matrix, usually after mean centering the data for each attribute. A relevant previous work uses PCA over an initial set of grasping configurations to find a bidimensional grasp subspace [6], and further works have used this subspace to look for static grasping configurations [7], [8]. 


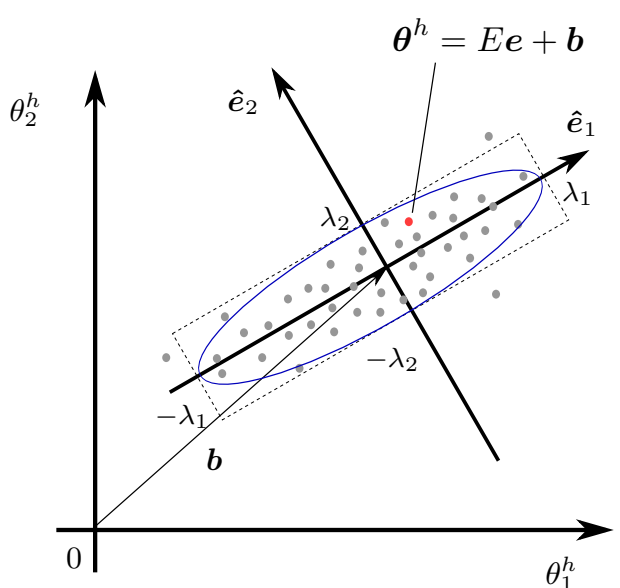

Fig. 1. Mappping from the hand configuration space $\mathcal{C}^{\text {h }}$ to the principal motion direction space $\mathcal{E}$.

The result of the PCA over the set of samples is the PMD space $\mathcal{E}$, which is basically a new reference frame located at the mean center value $\mathbf{b}$ (see Fig. 1). The PMDs define a base of $\mathcal{E}$, namely the columns of a rotation matrix $E=\left(\hat{\boldsymbol{e}}_{1}, \hat{\boldsymbol{e}}_{2}, \ldots, \hat{\boldsymbol{e}}_{H}\right)$, where $H$ is the dimension of the configuration space of the hand. Thus, the hand workspace $W^{H}$ is an axis-aligned box in $\mathcal{E}$ centered at $\mathbf{b}$ and with the size, $\lambda_{i}$, of each side chosen proportional to the standard deviation of the samples along $\hat{\boldsymbol{e}}_{i}$, such that the $W^{H}$ contains around the $95 \%$ of the samples.

\section{B. Probabilistic Roadmaps}

Probabilistic roadmaps (PRMs) are sampling-based path planners that randomly generate collision-free samples of configuration space $(\mathcal{C})$ and connect them with free paths capturing the connectivity of the free space by forming graphs called roadmaps [2]. These planners are giving very good results, being its success mainly due to its samplingbased nature, i.e. they do not require the explicit characterization of the obstacles of $\mathcal{C}$ and its efficiency relies on the sample set. Therefore, the generation of samples is one of the crucial factors in the performance of these planers.

Probabilistic roadmaps are demonstrated to be probabilistic complete, e.g. for the basic PRM method the number of samples necessary to achieve a probability of failure below a given threshold has been determined [9]. For difficult pathplanning problems, however, like those involving narrow passages or high degrees of freedom robots in cluttered environments, this number might be quite large and, therefore, importance sampling or dimension-reduction techniques have been introduced (e.g. [10], [11]).

The use of a PRM for the planning of anthropomorphic hand-arm systems based on the reduction of the sampling space using the principal motion directions was first proposed by the authors in [3]. Following this line, the present paper focuses on the sampling process by proposing a more efficient obtention of samples. The main features of the developed PRM, that improve the approach presented in [3], are: a) several hand configurations are assigned to each arm configuration; b) hand configurations are obtained

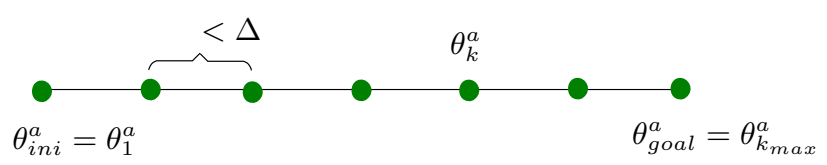

Fig. 2. Arm configurations $\boldsymbol{\theta}_{k}^{a}$, equally spaced a distance below a given threshold $\Delta$, along the rectilinear path joining $\boldsymbol{\theta}_{i n i}^{a}$ and $\boldsymbol{\theta}_{\text {goal }}^{a}$.

from a subspace $\mathcal{S C}^{\mathrm{h}}$ of the hand configuration space $\mathcal{C}^{\mathrm{h}}$ that, by construction, contains both the initial and the goal configurations, solving in this way the problem of connecting them to this subspace; c) the dimension of this subspace is not fixed but is iteratively increased, as required by the difficulty of the task at hand; d) the direction of the first dimension of $\mathcal{S C}^{\mathrm{h}}$ is defined to always connect the initial and goal hand configurations.

\section{Problem Statement AND PRoposed Solution}

Given the initial and the goal configurations of the handarm system $\boldsymbol{\theta}_{i n i}=\left(\boldsymbol{\theta}_{\text {ini }}^{h}, \boldsymbol{\theta}_{\text {ini }}^{a}\right)$ and $\boldsymbol{\theta}_{\text {goal }}=\left(\boldsymbol{\theta}_{\text {goal }}^{h}, \boldsymbol{\theta}_{\text {goal }}^{a}\right)$, with $\boldsymbol{\theta}_{\text {ini }}^{h}$ and $\boldsymbol{\theta}_{\text {goal }}^{h}$ being the configurations of the hand, and $\boldsymbol{\theta}_{i n i}^{a}$ and $\boldsymbol{\theta}_{\text {goal }}^{a}$ those of the arm, the problem to be solved is the search of an anthropomorphic-like collision-free path connecting them.

It is assumed that $\boldsymbol{\theta}_{\text {goal }}$ is a grasp or preshape configuration that can be reached from $\boldsymbol{\theta}_{i n i}$ by a simple arm motion coordinated with the proper hand motions needed to avoid collisions with the obstacles. Following this rationale, the proposed approach is based on the decomposition of the motions between those of the arm and those of the hand, and then looking for a common solution using a unique probabilistic roadmap planner. Moreover, hand motions are computed in a reduced search space using some principal motion directions that, besides reducing the number of DOF, attempt to lead the mechanical hand movements through anthropomorphic postures.

Section III-A presents the main algorithm (FIND PATH) to construct the PRM and search for the solution path. This algorithm makes use of the HAND MOTION algorithm, introduced in Section III-B, to obtain hand configurations using the reduced search space. Starting with one dimension, this algorithm is iteratively called with an increasing dimension until FIND PATH either returns a solution path, or a failure flag if no path is found and the dimension of the search space has reached that of the hand configuration space.

\section{A. Main algorithm}

Given the initial and the goal configurations of the handarm system $\boldsymbol{\theta}_{i n i}=\left(\boldsymbol{\theta}_{i n i}^{a}, \boldsymbol{\theta}_{i n i}^{h}\right)$ and $\boldsymbol{\theta}_{\text {goal }}=\left(\boldsymbol{\theta}_{\text {goal }}^{a}, \boldsymbol{\theta}_{\text {goal }}^{h}\right)$, the following algorithm searches for an obstacle-free path connecting them. The algorithm works with normalized joint values in the range $[0,1]$, and thus in an adimensional configuration space.

\section{Algorithm FIND PATH $\left(\boldsymbol{\theta}_{\text {ini }}, \boldsymbol{\theta}_{\text {goal }}\right)$}

1) Generate arm configurations $\boldsymbol{\theta}_{k}^{a}$, equally spaced a distance below a given threshold $\Delta$, along the rectilinear path joining $\boldsymbol{\theta}_{\text {ini }}^{a}$ and $\boldsymbol{\theta}_{\text {goal }}^{a}$ (Fig. 2). If $k_{\text {max }}$ is the 

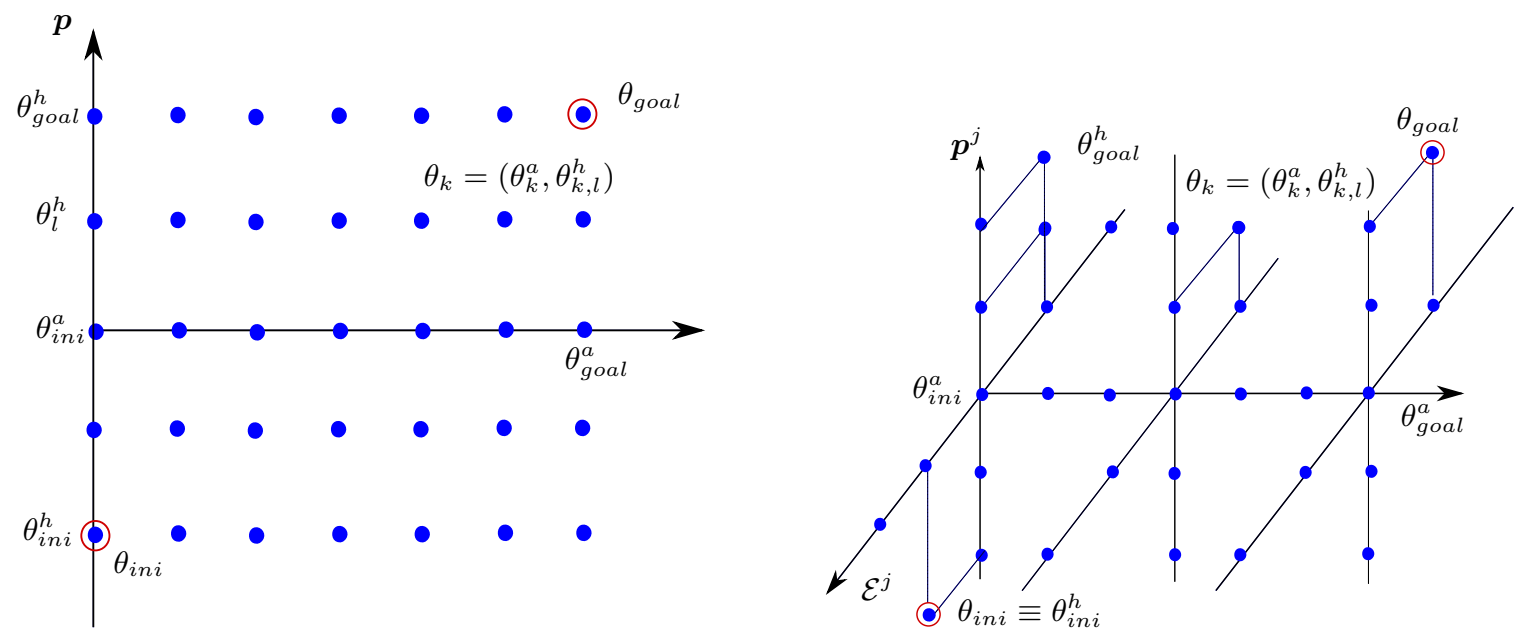

Fig. 3. Hand-arm configurations. Arm configurations are represented on the horizontal axis that corresponds to the direction connecting $\boldsymbol{\theta}_{i n i}^{a}$ and $\boldsymbol{\theta}_{\text {goal }}^{a}$. For $j=1$ (left figure), hand configurations are represented by the vertical axis that corresponds to the direction $\boldsymbol{p}$ connecting $\boldsymbol{\theta}_{\text {ini }}^{h}$ and $\boldsymbol{\theta}_{\text {goal }}^{h}$. For $j=2$ (right figure) they are represented by the plane defined by $\mathcal{E}^{j}$ and $\boldsymbol{p}^{j}$.

resulting number of configurations to be generated (due to the distance between $\boldsymbol{\theta}_{i n i}^{a}$ and $\boldsymbol{\theta}_{i n i}^{a}$ ), then:

$\boldsymbol{\theta}_{k}^{a}=\left(\boldsymbol{\theta}_{\text {goal }}^{a}-\boldsymbol{\theta}_{\text {ini }}^{a}\right) \frac{k-1}{k_{\max }-1}+\boldsymbol{\theta}_{\text {ini }}^{a} \quad k=1, \ldots, k_{\text {max }}$

2) Create a PRM and look for a solution by iteratively increasing the number $j$ of DOF used to generate the hand configurations (see Fig. 3 for $j=1$ and $j=2$ ). For $j=1$ to $H$ do:

a) Generate a set $S$ with $N$ hand configurations.

i) With the arm at $\boldsymbol{\theta}_{\text {ini }}^{a}$, generate $N / 2$ hand configurations using the HAND MOTION algorithm with $j$ DOF, such that the hand-arm system is collision-free.

ii) With the arm at $\boldsymbol{\theta}_{\text {goal }}^{a}$, generate $N / 2$ hand configurations using the HAND MOTION algorithm with $j$ DOF, such that the hand-arm system is collision-free.

b) For $k=0$ to $k_{\max }$ do:

With the arm at $\boldsymbol{\theta}_{k}^{a}$, locate the hand at each configuration of $S$ and test whether the hand-arm system is collision-free.

i) If it is collision-free, add the hand-arm configuration as a roadmap node.

ii) Otherwise, generate both a random hand configuration using the HAND MOTION algorithm with $j \mathrm{DOF}$, and a random configuration of the arm on a small neighborhood around $\boldsymbol{\theta}_{k}^{a}$ (defined by a fixed radius $\rho$ ).

Check if the resulting hand-arm configuration is collision-free. Add the hand-arm configuration as a roadmap node if this is the case, or keep trying until a collision-free one is found (up to a maximum number $T$ of trials).

c) Complete the roadmap by connecting neighboring nodes using a simple straight-line local planner. d) Search for PATH in the roadmap to connect $\boldsymbol{\theta}_{\text {ini }}$ and $\boldsymbol{\theta}_{\text {goal }}$. Return PATH, if found.

\section{3) Return FAILURE}

\section{B. Hand motion algorithm}

The generation of the hand configurations used in the main algorithm is done in the following way given the desired dimension $j$ of the search space.

Algorithm HAND MOTION $\left(\boldsymbol{\theta}_{\text {ini }}^{h}, \boldsymbol{\theta}_{\text {goal }}^{h}, E, \boldsymbol{b}, j\right)$

1) Obtain the initial and final configurations of the hand expressed in terms of the PMDs in $\mathcal{E}$, i.e.:

$$
\begin{gathered}
\boldsymbol{e}_{i n i}=E^{-1}\left(\boldsymbol{\theta}_{i n i}^{h}-\boldsymbol{b}\right) \\
\boldsymbol{e}_{\text {goal }}=E^{-1}\left(\boldsymbol{\theta}_{\text {goal }}^{h}-\boldsymbol{b}\right)
\end{gathered}
$$

2) Find a nominal direct path between $\boldsymbol{e}_{i n i}$ and $\boldsymbol{e}_{\text {goal }}$. Let $\boldsymbol{e}=\boldsymbol{e}_{i n i}+\alpha\left(\boldsymbol{e}_{\text {goal }}-\boldsymbol{e}_{i n i}\right)$ be the straight line in $\mathcal{E}$ defined by $\boldsymbol{e}_{i n i}$ and $\boldsymbol{e}_{\text {goal }}$.

Find the intersection points of this line with the hyperplanes containing each face of the axis-aligned box $W^{H}$ defining the hand work-space in $\mathcal{E}\left(W^{H}\right.$ was defined in subsection II-A; see Figure 1 for a qualitative 2D illustration). This is simply done by solving $2 H$ linear equations of first order. Since there are $H=11$ PMDs and $\boldsymbol{e}_{i n i}$ and $\boldsymbol{e}_{\text {goal }}$ lie inside the hand workspace, there are 22 intersection points, 11 of them defined by negative values of $\alpha$ and the other 11 defined by positive values of $\alpha$.

Let $\boldsymbol{e}_{\min }$ and $\boldsymbol{e}_{\max }$ be the points defined, respectively, by the greatest negative and the smaller positive values of $\alpha, P$ be the segment delimited by $\boldsymbol{e}_{\min }$ and $\boldsymbol{e}_{\max }, \boldsymbol{e}_{\operatorname{mid}}$ the middle point of $P$, and $\boldsymbol{p}=\left(\boldsymbol{e}_{\max }-\boldsymbol{e}_{\min }\right) / 2$.

3) Re-order the vectors $\hat{\boldsymbol{e}}_{i}$ of $E$ (Section II-A) according to the size of the components of $\boldsymbol{p}$ along them, from the smallest to the largest one. This is done using an 


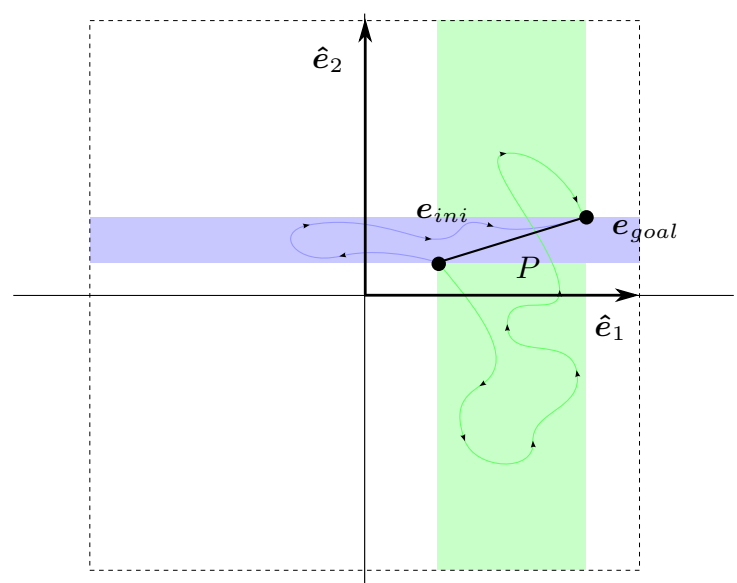

Fig. 4. Illustration of the search region in a $2 \mathrm{D}$ example given $\hat{e}_{1}, \hat{\boldsymbol{e}}_{2}$ and $\boldsymbol{p}$. The blue (dark gray) region is generated sweeping along $\hat{\boldsymbol{e}}_{1}$ the projection of $P$ onto $\hat{\boldsymbol{e}}_{2}$, and green region (light gray) region is generated sweeping along $\hat{\boldsymbol{e}}_{2}$ the projection of $P$ onto $\hat{\boldsymbol{e}}_{1}$. Since $\left|\boldsymbol{p} \cdot \hat{\boldsymbol{e}}_{2}\right|<\left|\boldsymbol{p} \cdot \hat{\boldsymbol{e}}_{1}\right|$ the green region is larger that the blue one, so it is more likely to find a path in the former.

ordering matrix $R$ to update $E$ as

$$
E \leftarrow R E \quad \text { and } \quad \boldsymbol{b} \leftarrow R \boldsymbol{b}
$$

such that the resulting vectors $\hat{\boldsymbol{e}}_{i}$ of the basis $E=\left(\hat{\boldsymbol{e}}_{1}, \ldots, \hat{\boldsymbol{e}}_{i}, \ldots, \hat{\boldsymbol{e}}_{n}\right) \quad$ satisfy $\left|\boldsymbol{p} \cdot \hat{\boldsymbol{e}}_{i}\right| \leq\left|\boldsymbol{p} \cdot \hat{\boldsymbol{e}}_{k}\right| \forall i<k$.

The reason for this order is that the vectors $\hat{\boldsymbol{e}}_{i}$ will be iteratively used to increase the dimension of the search space, and using first the vectors $\hat{e}_{i}$ with smaller components along the direction of $\boldsymbol{p}$ will likely produce a larger search region and therefore increase the likelihood of finding a solution, as illustrated in Fig. 4 for a $2 \mathrm{D}$ case. It should be noted that this is an heuristic step, since there is no guarantee that the order of vectors $\hat{\boldsymbol{e}}_{i}$ actually allow finding a solutions with smaller number of iterations (on the other hand, the size of $W^{H}$ along each vector $\hat{\boldsymbol{e}}_{i}$ suggests another possible useful order).

4) Generate the sampling subspace of dimension $j$ as a subspace of the hand workspace $W^{H}$ :

a) Generate two subspaces of $\mathcal{E}$, one called $\mathcal{E}^{j}$ defined by the first $j-1$ elements of $E$ i.e. $E^{j}=\left(\hat{e}_{1}, \ldots, \hat{\boldsymbol{e}}_{j-1}\right)$ is a basis of $\mathcal{E}^{j}$, and the other subspace called $\mathcal{E}^{j \perp}$ defined by the remaining $n-j+1$ elements of $E$, i.e. $E^{j \perp}=\left(\hat{e}_{j}, \ldots, \hat{e}_{n}\right)$ is a basis of $\mathcal{E}^{j \perp}$.

b) Project $\boldsymbol{p}$ and $\boldsymbol{e}_{\text {mid }}$ on $\mathcal{E}^{j \perp}$, and let $\boldsymbol{p}^{j}$ and $\boldsymbol{e}_{\text {mid }}^{j}$ be the resultant vector and point, respectively.

c) Obtain the sampling subspace $\mathcal{S C}^{j}$ defined by the orthogonal basis $S C^{j}=\left(E^{j}, \boldsymbol{p}^{j}\right)$.

Fig. 5 illustrates these steps for a hypothetical 3 -dimensional $\mathcal{E}$ for the first and second iteration (i.e. for $j=1$ and $j=2$ ).

5) Generate samples $e_{l}^{j} \in \mathcal{S C}^{j}, l=1, \ldots, l_{\text {max }}$ :

a) Generate $l_{\max }$ samples $\boldsymbol{s}_{l}$ in a space of dimension $j$ with each component in the range $[0,1]$. b) Map the samples $\boldsymbol{s}_{l}$ to $\mathcal{S C}^{j}$ as

$$
e_{l}^{j}=2 s_{l}-1
$$

Note that the samples could be generated directly in $\mathcal{S C}^{j}$ without using the auxiliar space of vectors $s_{l}$, but this option was preferred in order to allow future applications of deterministic sampling strategies on a regular sampling space.

6) Map the samples $\boldsymbol{e}_{l}^{j}$ to $\mathcal{E}$ as (Fig. 5)

$$
\boldsymbol{e}_{l}=S C^{j} \boldsymbol{e}_{l}^{j}+\boldsymbol{e}_{m i d}^{j}
$$

7) Map the samples $\boldsymbol{e}_{l}$ to the hand work-space expressed in the joint coordinates, which is done as

$$
\boldsymbol{\theta}_{l}^{h}=E \boldsymbol{e}_{l}+\boldsymbol{b}
$$

Thus, the hand joint values ${ }^{1}$ are obtained from the initial samples $s_{l}$ as

$$
\boldsymbol{\theta}_{l}^{h}=E\left(S C^{j}\left(2 \boldsymbol{s}_{l}-1\right)+\boldsymbol{e}_{m i d}^{j}\right)+\boldsymbol{b}
$$

In order to facilitate the understanding, the algorithm describes the whole procedure to generate the samples of the hand configuration, but it must be noted that Steps 1 to 3 do not need to be repeated each time new samples need to be generated for the same task.

\section{IMPLEMENTATION AND EXAMPLES}

The proposed approach has been implemented in $\mathrm{C}$ language and tested on a simulator considering the $\mathrm{SAH}$ hand, with 13 DOF, mounted on a 3 DOF robot arm. Two scenarios have been defined, both sharing the same initial and goal hand configurations $\boldsymbol{\theta}_{i n i}^{h}$ and $\boldsymbol{\theta}_{\text {goal }}^{h}$, respectively. Some snapshots of hand postures along the motion direction $p$ connecting $\boldsymbol{\theta}_{i n i}^{h}$ and $\boldsymbol{\theta}_{\text {goal }}^{h}$ can be seen in Fig. 6; and those corresponding to a linear combination of $\boldsymbol{p}^{2}$ and $e_{1}$ are illustrated in Fig. 7.

The variable of the algorithm FIND PATH $\left(\boldsymbol{\theta}_{\text {ini }}, \boldsymbol{\theta}_{\text {goal }}\right)$ have been set to the following values:

- $\Delta=0.04$, the distance between arm configurations.

- $N=8 j$, the number of samples of the set $S$ of hand configurations, with $\mathrm{j}$ the number of DOF used in the HAND MOTION algorithm.

- $\rho=0.05$, the radius defining a small neighborhood around arm configurations.

- $T=100$, the maximum number of trials to obtain a collision-free hand-arm configuration.

Figures 8 and 9 show snapshots of the solution paths found, respectively, for the first (less constrained) and the second (more constrained due to a larger obstacle bar) scenario. In both cases, the number of arm configurations considered along the straight path connecting $\boldsymbol{\theta}_{i n i}^{h}$ and $\boldsymbol{\theta}_{\text {goal }}^{h}$ resulted $k_{\max }=8$. The solution to the first one was found sampling the hand configurations only along $\boldsymbol{p}$. The averaged computation time over several runs was $3.2 \mathrm{~s}$ on a PC 3.0 $\mathrm{GHz}$, and the PRM were composed of a mean of 168 nodes.

\footnotetext{
${ }^{1}$ Recall that these are normalized values, i.e. in the range $[0,1]$. The joint angles are then obtained by denormalizing using the joint ranges.
} 


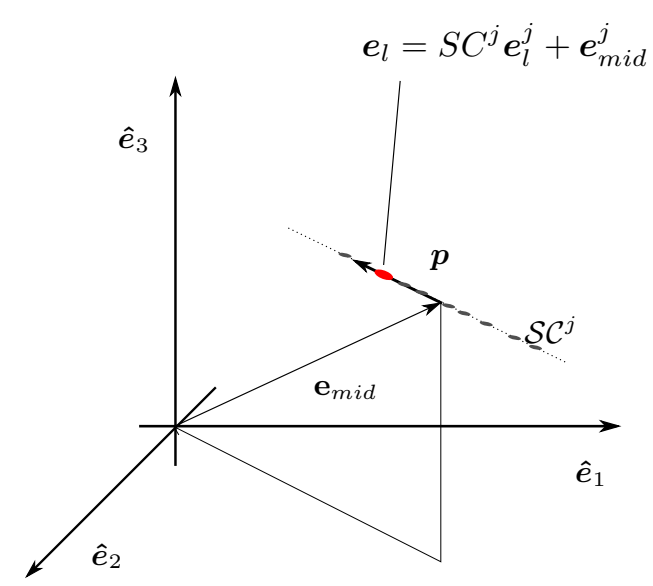

a) $j=1$

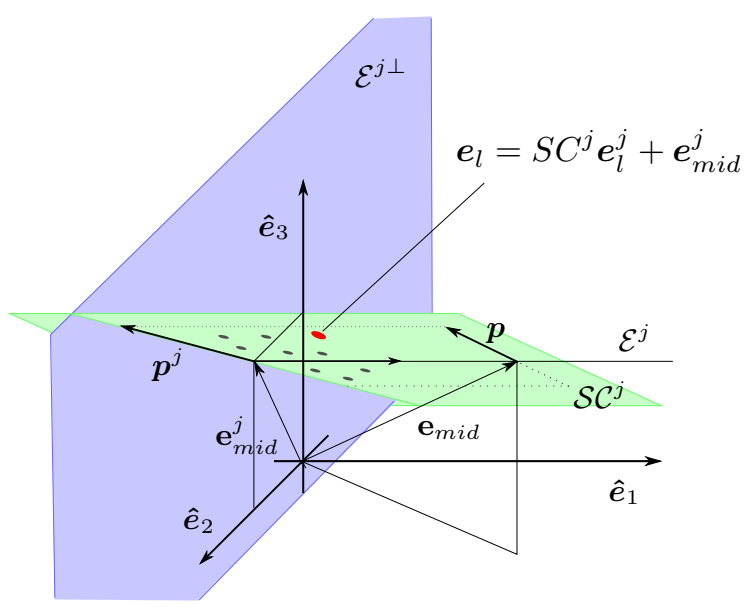

b) $j=2$

Fig. 5. Illustration of the HAND MOTION algorithm for a hypothetical 3-dimensional $\mathcal{E}$ : a) First iteration, $j=1$, showing the direct path in the space $\mathcal{S C}^{1}$ defined by $\boldsymbol{p}$; b) Second iteration, $j=2$, showing the path in the space $\mathcal{S} \mathcal{C}^{2}$ defined by $\hat{\boldsymbol{e}}_{1}$ and $\boldsymbol{p}^{2} \in \mathcal{E}^{2 \perp}$.
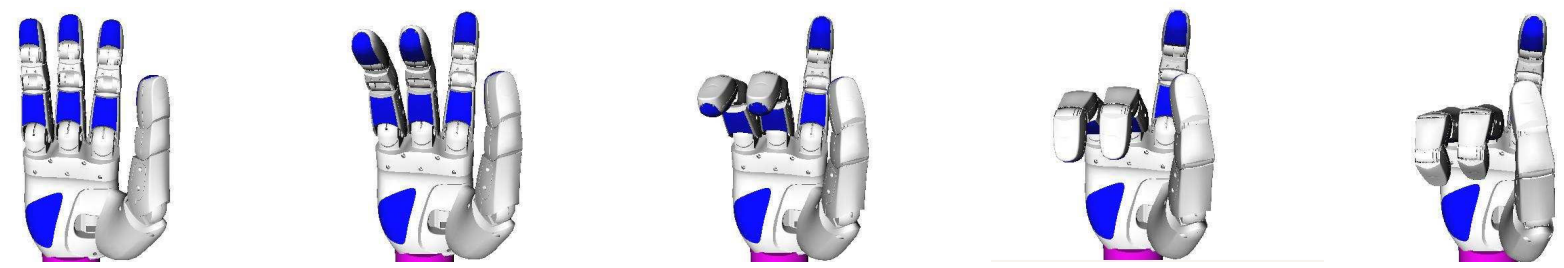

Fig. 6. Snapshots of hand postures along the motion direction $\boldsymbol{p}$ connecting $\boldsymbol{\theta}_{i n i}^{h}$ and $\boldsymbol{\theta}_{\text {goal }}^{h}$.

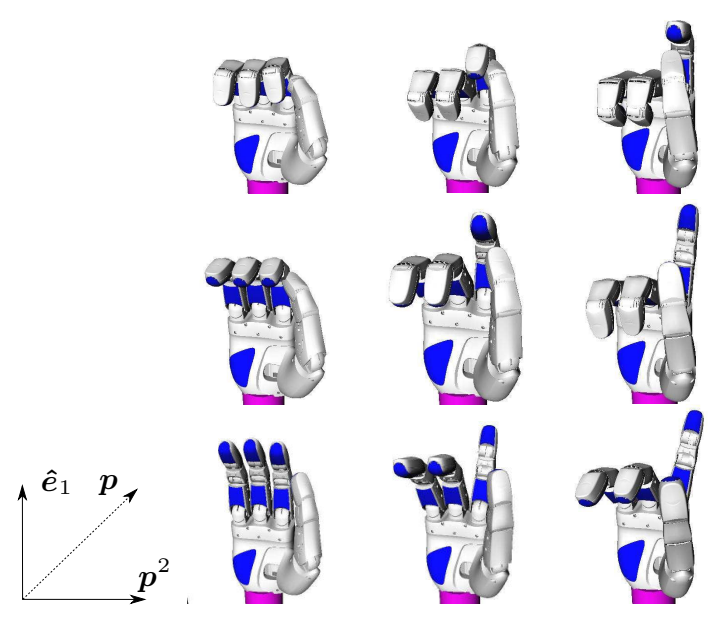

Fig. 7. Snapshots of hand postures corresponding to a linear combination of directions $\boldsymbol{p}^{2}$ (horizontal) and $\hat{\boldsymbol{e}}_{1}$ (vertical), considered along all their ranges.

The solution to the second scenario was found sampling the hand configurations along the subspace defined by $\boldsymbol{p}^{2}$ and $\hat{e}_{1}$ (note that in this scenario, the sole motion along $\boldsymbol{p}$ cannot produce the necessary flexion of the index finger in order to avoid a collision with the obstacle bar - Fig. 9d). The averaged computation time over several runs was $6.5 \mathrm{~s}$ and the PRM were composed of a mean of 205 nodes. In both scenarios those configurations closer to the obstacle bars needed resampling (step 2.b.ii of the FIND PATH algorithm).

\section{DISCUSSION AND CONCLUSIONS}

An approach to compute the movements of an anthropomorphic hand mounted on a robot arm has been presented in this work. The proposed approach is intended to be particularly useful in the final part of a hand-arm movement, close to the grasp action itself or to a hand-object interaction, i.e. when the existence of a free path for the arm considering a bounding box for the hand is unlikely to be found, and therefore the movements should be planned in a high dimensional space (defined by the hand-arm degrees of freedom) requiring a lot of computation.

The key point of the proposed approach is that the search of a solution path for the hand-arm system is initially done considering movements of the hand in a 1-dimensional subspace, and only if no solution is found the dimension of the search space is iteratively increased. Besides, by construction, the initial and goal hand-arm configuration always belong to the search space.

The approach has been implemented and good solutions were obtained in simulation for the SAH hand, with $13 \mathrm{DOF}$, mounted on a 3 DOF robot arm. Nevertheless, the approach is of heuristic nature and cannot guarantee an optimal performance. If the solution is found after a significative number of iteration the computational cost may be larger than looking for the solution directly in the search space defined by all 


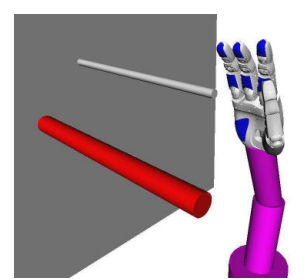

a)

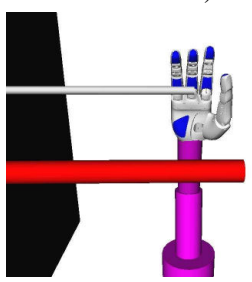

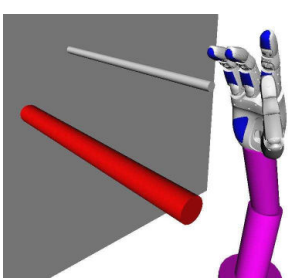

b)

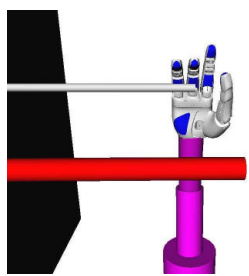

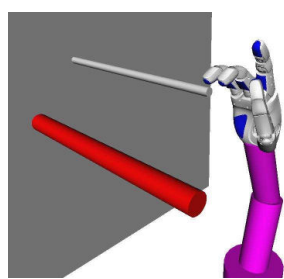

c)

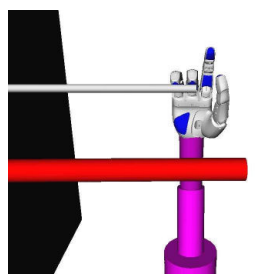

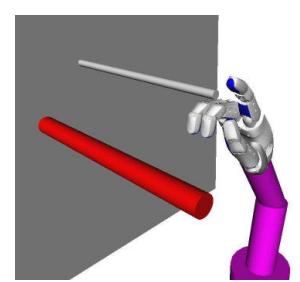

d)

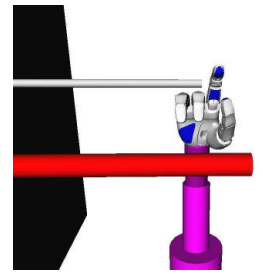

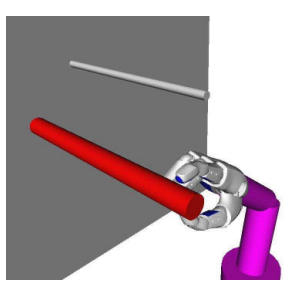

e)

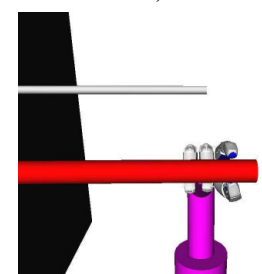

Fig. 8. Snapshots of a successful attempt to connect $\boldsymbol{\theta}_{i n i}$ and $\boldsymbol{\theta}_{\text {goal }}$ on the first scenario (see the accompanying video for the whole sequence).

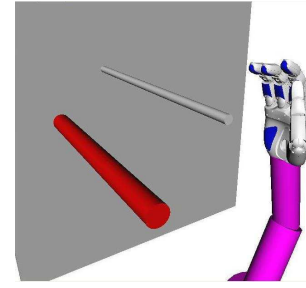

a)

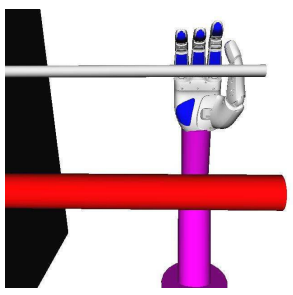

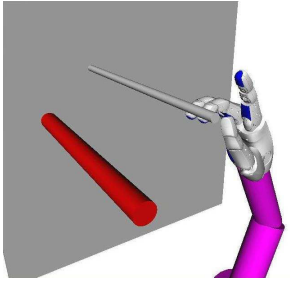

b)

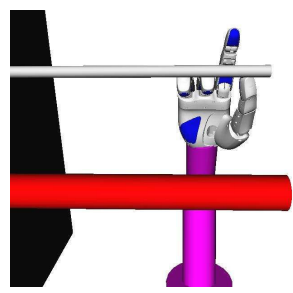

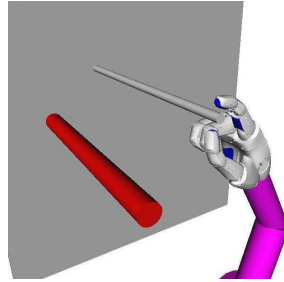

c)

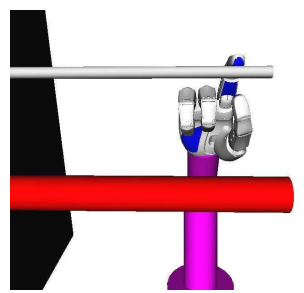

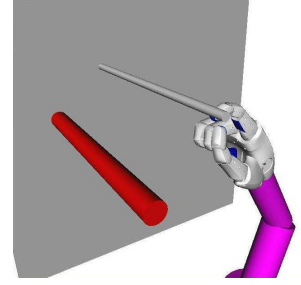

d)

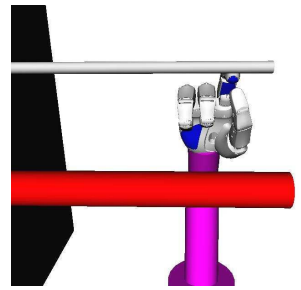

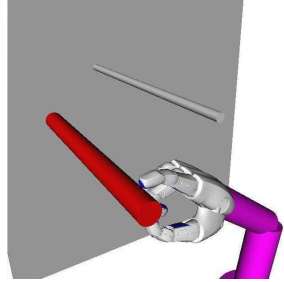

e)

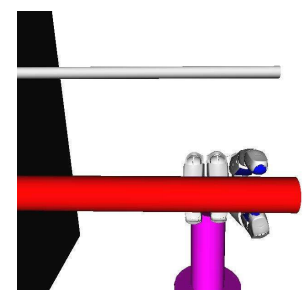

Fig. 9. Snapshots of a successful attempt to connect $\boldsymbol{\theta}_{i n i}$ and $\boldsymbol{\theta}_{\text {goal }}$ on the second scenario (see the accompanying video for the whole sequence).

the DOF of the hand-arm system. Nevertheless, the approach seems to be efficient for everyday hand movements, avoiding complicate and unusual hand configurations, and opens a new direction for future improvements. One potential source of performance improvement is the selection of a "good" sequence of PMDs in the iterative dimensionality increase. We used a particular ordering criterion here but we are aware that some others producing different sequences also make sense, thus, exhaustive experimentation should be done to find out the best criterion. Other topics that deserve further research in order to improve the generation of the unique PRM are the influence of the variables of the algorithm and the way in which the samples of the hand configuration are replicated for each arm configuration.

\section{REFERENCES}

[1] S. M. LaValle, Planning Algorithms. Cambridge University Press, 2006.

[2] L. E. Kavraki, P. Svestka, J.-C. Latombe, and M. K. Overmars, "Probabilistic roadmaps for path planning in high - dimensional configuration spaces," IEEE Trans. on Robotics and Automation, vol. 12, no. 4, pp. 566-580, August 1996.
[3] J. Rosell, R. Suárez, C. Rosales, J. A. García, and A. Pérez, "Motion planning for high DOF anthropomorphic hands," in Proc. of the IEEE Int. Conf. on Robotics and Automation, 2009, pp. 4025-4030.

[4] I. T. Jolliffe, Principal Component Analysis. Upper Saddle River, NJ, USA: Springer Series in Statistics, 2002.

[5] Schunk GmbH \& Co. KG, "Schunk anthropomorphic hand," http://www.schunk.com/, May 2006.

[6] M. Santello, M. Flanders, and J. F. Soechting, "Postural hand synergies for tool use," Journal of Neuroscience, vol. 18, no. 23, pp. $10105-$ 10115 , December 1998.

[7] M. T. Ciocarlie and P. K. Allen, "Hand posture subspaces for dexterous robotic grasping," The International Journal of Robotics Research, vol. 28, no. 7, pp. 851-867, July 2009.

[8] A. Tsoli and O. C. Jenkins, "2D Subspaces for user-driven robot grasping," in Proc. of the RSS 2007 Workshop on Robot Manipulation: Sensing and Adapting to the Real World, 2007.

[9] L. E. Kavraki, M. N. Kolountzakis, and J.-C. Latombe, "Analysis of probabilistic roadmaps for path planning," IEEE Trans. on Robotics and Automation, vol. 14, no. 1, pp. 166 -171, Feb. 1998.

[10] V. Boor, M. H. Overmars, and A. F. van der Stappen, "The Gaussian sampling strategy for probabilistic roadmap planners," in Proc. of the IEEE Int. Conf. on Robotics and Automation, 1999, pp. 1018-1023.

[11] A. Rodríguez, A. Pérez, J. Rosell, and L. Basañez, "Sampling-based path planning for geometrically-constrained objects," in Proc. of the IEEE Int. Conf. on Robotics and Automation, 2009, pp. 2074-2079. 